\title{
Cerebroplacental ratio for prediction of adverse intrapartum and neonatal outcomes in a term uncomplicated pregnancy
}

\author{
Mariam Lotfy Mohamed, Salwa Adel Mohamed* (1) and Amal Mohamed Elshahat
}

\begin{abstract}
Background: Fetal hypoxia is one of the major causes of high perinatal morbidity and mortality rates. Doppler ultrasound tests such as cerebroplacental ratio (CPR) evaluation are commonly used to assess blood flow disturbances in placento-umbilical and feto-cerebral circulations. A low cerebroplacental ratio has been shown to be associated with an increased risk of stillbirth regardless of the gestation or fetal weight. We conducted this study to assess the fetal cerebroplacental ratio in prediction of adverse intrapartum and neonatal outcomes in a term, uncomplicated pregnancy to reduce fetal and neonatal morbidity and mortality.

Results: It was found that neonates with CPR $\leq 1.1$ had significantly higher frequencies of cesarean delivery (CS) for intrapartum fetal compromise compared to those with CPR $>1.1(p=0.043)$. Neonates with $C P R \leq 1.1$ had significantly lower Apgar score at $1 \mathrm{~min}$ and $5 \mathrm{~min}$ than those with $\mathrm{CPR}>1.1(p=0.004)$ and $(p=0.003)$, respectively. Neonates with $C P R \leq 1.1$ had significantly higher rates of NICU admission than those with CPR $<1.1 \quad(p=0.004)$.

Conclusion: The cerebroplacental ratio shows the highest sensitivity in the prediction of fetal heart rate abnormalities and adverse neonatal outcome in uncomplicated pregnancies at term. The cerebroplacental ratio index is useful in clinical practice in antenatal monitoring of these women in order to select those at high risk of intra- and postpartum complications.
\end{abstract}

Keywords: Cerebroplacental ratio, Perinatal outcome, Term pregnancy

\section{Background}

Fetal hypoxia is one of the major causes of high perinatal morbidity and mortality rates. It is a major contributor to adverse perinatal outcomes, including stillbirth, hypoxic-ischaemic brain injury. It may also lead to various neurodevelopmental disabilities [1].

Labor is an asphyxial process, with contractions reducing blood flow in the uterine arteries and thus decreasing oxygen availability to the placenta and fetus. This results in a gradual deterioration of the fetal condition reflecting a steady decline in the ability of the placenta to oxygenate the fetus as labor progresses [2]. The fetus responds

*Correspondence: salwa.adel@med.suez.edu.eg

Department of Obstetrics and Gynecology, Faculty of Medicine, Suez

Canal University, Ismailia, Egypt to uterine contractions with acute cerebral redistribution, evidenced by a reduced middle cerebral artery pulsatility index [3].

Most cases of asphyxia during labor occur due to a gradual decline in the ability of the fetus to tolerate the process of parturition. It is likely that these infants have decreased fetoplacental reserve prior to the onset uterine contractions [4]. If delivery is not expedited, these infants are at a serious risk of brain injury and subsequent permanent disability with hypoxic-ischaemic encephalopathy, a key risk factor for the development of cerebral palsy in term infants [5].

These compromised fetuses frequently require rapid delivery by emergency operative delivery that carries more maternal risk than less urgent procedures. Neonatal 
outcomes are also significantly poorer following emergency caesarean for fetal distress [6].

Fetal hypoxia activates a number of defense mechanisms, such as modification of fetal heart rate (FHR), increase in blood pressure, and redistribution of the blood to the heart, brain, and adrenal glands. Low oxygen partial pressure $\left(\mathrm{pO}_{2}\right)$ leads to cerebral vasodilation and a fall in vascular resistance, which results in a decrease in middle cerebral artery resistance index (MCA RI) values [7].

The brain sparing (BS) phenomenon is considered as an adaptive mechanism of the fetus which is activated to protect the fetal brain in adverse conditions. The phenomenon does not completely protect the brain against the effects of hemodynamic changes. Long-term studies have shown that children with BS have lower IQ scores, impaired cognitive function, attention deficit, and greater difficulties at school [8].

The most widely used screening method for intrapartum hypoxia is electronic fetal heart rate monitoring. It has very poor specificity and positive predictive value for hypoxia and adverse neonatal outcome and may indeed be considered a failure of public health policy [9].

The current challenge is therefore to identify vulnerable fetuses at risk of hypoxic complications, particularly within an apparently low-risk cohort before the onset of labor.

Doppler ultrasound tests such as cerebroplacental ratio (CPR) evaluation are commonly used to assess blood flow disturbances in placento-umbilical and feto-cerebral circulations [10].

The fetal cerebroplacental ratio is the ratio of the middle cerebral artery pulsatility index to the umbilical artery pulsatility index [11]. The cerebroplacental ratio evaluation is used to detect and assess the fetal response to oxygen deficiency in utero. With the progress of pregnancy, the resistance in fetal circulation decreases gradually. Nevertheless, the values of middle cerebral artery pulsatility index (MCA PI) should remain higher than umbilical artery pulsatility index (UA PI) values, which implicates that CPR, being the ratio of (MCA PI) to (UA $\mathrm{PI})$, should be higher than (1-1.1) in uncomplicated pregnancies [12].

In some term small for gestational age fetuses, the middle cerebral artery pulsatility index is reduced despite normal umbilical artery Doppler indices, and this is associated with poorer perinatal outcomes and adverse neurobehavior sequelae [13].

A low cerebroplacental ratio has been shown to be associated with an increased risk of stillbirth regardless of the gestation or fetal weight [14].

So in the current work, the cerebroplacental ratio will be assessed to identify fetuses that fail to reach their genetic growth potential and/or are at increased risk of perinatal complications.

\section{Methods}

This was a prospective observational cross-sectional study conducted at the outpatient clinic of the Obstetrics and Gynecology Department, Suez Canal University Hospitals, from August 2018 till June 2019 on one hundred fifty primiparous women aged between 18 and 35 in uncomplicated singleton pregnancies, between 37 and 40 completed weeks with cephalic presentation to assess the fetal cerebroplacental ratio in prediction of adverse intrapartum and neonatal outcomes in a term, uncomplicated pregnancy. Table 1 shows the exclusion criteria that include multiple pregnancy, medical or surgical conditions with pregnancy, fetal congenital anomaly, fetal growth restriction, and premature rupture of membrane. During the study period, 173 women consented to participate in the study, of which 23 women were excluded for various reasons. These include 14 who underwent elective CS, 9 women who withdrawn from the study and did not have intrapartum fetal monitoring. Thus, the final study cohort compromise of 150 women (Fig. 1).

This study was carried out after gaining approval of the ethical committee in the Faculty of Medicine, Suez Canal University, and approval of the Suez Canal University Hospital administration board. An informed consent about participation in the study was thoroughly discussed with patients in an easy understandable language. Only consenting patients were included in the study. Patients were assured that they have the full right to refuse participation in the study or withdraw at any time and that refusal or withdrawal will not affect the quality of care

Table 1 Baseline characteristics of the studied sample

\begin{tabular}{ll}
\hline Variables & $\boldsymbol{n}=\mathbf{1 5 0}$ \\
\hline Age (years), mean \pm SD & $29.2 \pm 4.1$ \\
Occupation, $n$ (\%) & \\
$\quad$ Housewife & $80(53.3)$ \\
$\quad$ Employer & $40(26.7)$ \\
$\quad$ Manual worker & $30(20)$ \\
Residency, $n$ (\%) & \\
$\quad$ Rural & $88(58.7)$ \\
$\quad$ Urban & $62(41.3)$ \\
Socioeconomic status, $n(\%)$ & \\
$\quad$ Low & $65(43.3)$ \\
$\quad$ Middle & $68(45.3)$ \\
$\quad$ High & $17(11.3)$ \\
BMI (kg/m²), mean \pm SD & $22.4 \pm 1.4$
\end{tabular}

Data are presented as number (\%) or mean \pm standard deviation (SD) $B M I$ body mass index 


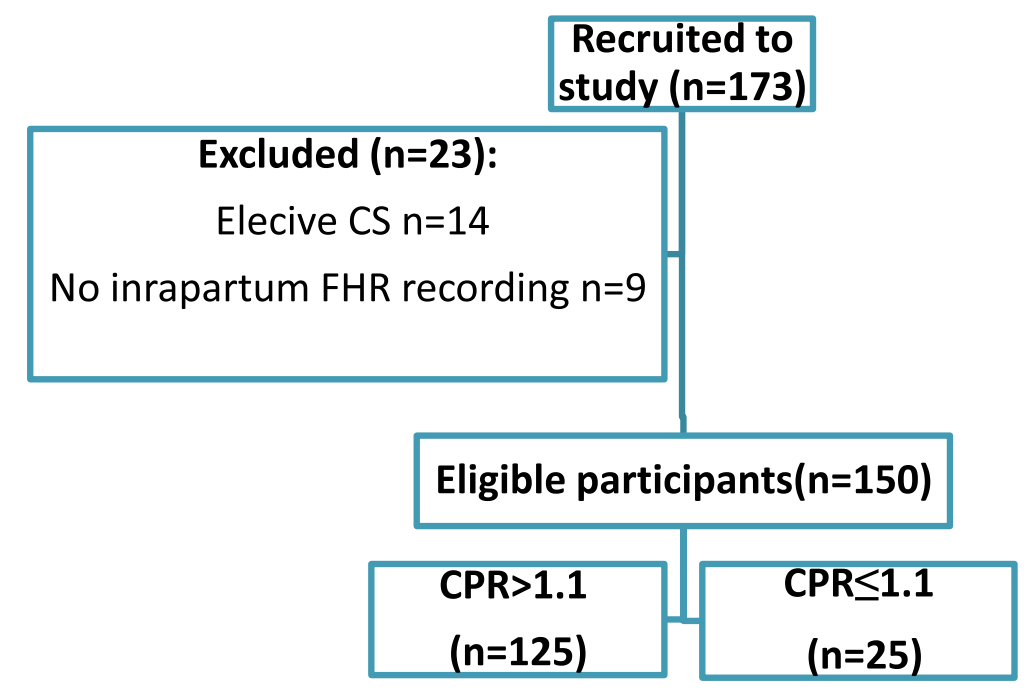

Fig. 1 Flowchart showing inclusion of the study participants. CPR cerebroplacental ratio, CS cesarean section

provided to them. Confidentiality of patient personal information will be maintained at all stages of the study.

At the first visit, all patients enrolled in the study were subjected to the following:

\section{History taking}

- Age, occupation, residence.

- Gestational age calculated by the last menstrual period.

- Past or current medical illness.

- History of any drug intake and allergy to any drugs.

- Smoking.

\section{Physical examination}

- General examination was done to exclude any disease or abnormality.

- Body mass index was calculated by dividing weight in kilogram/(height in meter) ${ }^{2}$.

- Blood pressure measurement.

\section{Ultrasound examination}

Gestational age was calculated based on the last menstrual period confirmed by a first-trimester ultrasound scan. All women underwent ultrasound assessments weekly, from 37 weeks until delivery including measurements of fetal biometry and fetal Dopplers (umbilical artery (UA) and fetal middle cerebral artery (MCA) pulsatility index (PI)). CPR was calculated at each attendance. All the measurements were performed in semi-recumbent position. The umbilical artery color Doppler waveform was collected from free floating parts of the umbilical cord during minimal fetal activity. For measurement of MCA, an axial view of fetal head was obtained at the level of cerebral peduncles, and then, the color Doppler was used to visualize the Circle of Willis and Doppler sample volume was placed on MCA that was easily identified on a major branch running anterolateral from the Circle of Willis toward the lateral edge of the orbit. The machine used was Mindray DC-60.

\section{Steps of the study}

At each weekly visit, the umbilical artery (UA) pulsatility index (PI) and middle cerebral artery (MCA) pulsatility index (PI) were calculated from three consecutive waveforms during fetal quiescence using an automated trace of the spectral Doppler waveform. The CPR was calculated as a simple ratio of the MCA PI to the umbilical artery PI. Values below 1.1 were reported as abnormal [12]. The last CPR prior to delivery is reported and used for all analyses.

$$
\mathrm{CPR}=\mathrm{MCA} \mathrm{PI} / \mathrm{UA} \mathrm{PI}
$$

Labor and delivery were managed in the institution. Participants and clinicians were blinded to ultrasound results, except in cases in which malpresentation, severe oligohydramnios (deepest vertical pocket $<1 \mathrm{~cm}$ ) or absent/reversed end-diastolic flow in the UA was detected. If any of these abnormalities was detected, obstetric caregivers were informed immediately, as these findings would alter clinical management.

Birth weight, mode and indication for birth, and neonatal outcomes including Apgar score and neonatal 
intensive care unit (NICU) admission were recorded within $48 \mathrm{~h}$ of birth. Decision for operative delivery for fetal compromise was recorded as that made by the treating obstetric team.

Pulsatility index in the middle cerebral, the umbilical artery, and the CPR ratio were evaluated weekly by Doppler ultrasonography, then were divided into two groups (according to last measurement): with CPR less than or equal to $1.1(n=25)$ and with CPR more than $1.1(n=$ 125), respectively. Abnormal flow indices were analyzed and compared to adverse pregnancy and neonatal outcome determinants.

\section{Statistical analysis}

Collected data will be processed using SPSS version 22 . Quantitative data will be expressed as mean \pm SD while qualitative data will be expressed as numbers and percentages. Student $t$ test will be used to test significance of difference for quantitative variables and chi-square will be used to test significance of difference for qualitative variables. A probability value ( $p$ value) $<0.05$ will be considered statistically significant. Data will be analyzed and appropriately presented in tables and graphs.

\section{Results}

The study revealed that neonates with CPR less than or equal to 1.1 were $16.7 \%$ and those with CPR more than 1.1forms $83.3 \%$.

There was no statistically significant association between CPR and diagnosis of oligohydramnios, absent/ reversed end-diastolic flow.

It was found that neonates with $\mathrm{CPR} \leq 1.1$ had significantly higher frequencies of CS delivery for intrapartum fetal compromise compared to those with CPR $>1.1$ $(p=0.043)$ and that neonates with CPR $\leq 1.1$ had significantly lower Apgar score at $1 \mathrm{~min}$ and $5 \mathrm{~min}$ than those with CPR $>1.1 \quad(p=0.004)$ and $(p=0.003)$, respectively. Neonates with $C P R \leq 1.1$ had significantly higher rates of NICU admission than those with CPR $>1.1(p=0.004)$

(Tables 2 and 3).

The ROC curve analysis of umbilical artery pulsatility index, fetal middle cerebral artery pulsatility index, and cerebroplacental ratio for prediction of different adverse intrapartum and neonatal outcomes shows that cerebroplacental ratio had the highest area under the curve (AUC) (0.72) for prediction of fetal heart rate (FHR) abnormality by cardiotocography (CTG) followed by fetal middle cerebral artery PI (0.65). Meanwhile, cerebroplacental ratio had the highest area under the curve (0.837) for prediction of CS delivery for intra-partum fetal compromise (IFC). For prediction of Apgar score $<7$ at $1 \mathrm{~min}$, both fetal middle cerebral artery pulsatility index $(0.65)$ and cerebroplacental ratio $(0.62)$ had the highest area under the curve with no a significant difference between them. While regarding CS delivery for IFC, cerebroplacental ratio had the highest area under the curve (0.72) followed by umbilical artery pulsatility index (0.66) (Figs. 2 and 3).

Cerebroplacental ratio was found to have the best accuracy in predicting CS delivery for IFC and the best accuracy in predicting Apgar score $<7$ at 1 min with sensitivity $60.89 \%$ and specificity $66.14 \%$. Similarly, cerebroplacental ratio was found to have the best accuracy in predicting NICU admission with sensitivity $63.16 \%$ and specificity 70.99\% (Tables 4 and 5)

\section{Discussion}

Intrapartum complications are a major contributor to adverse perinatal outcomes and significant neonatal morbidity [15]. The compromised fetuses frequently require rapid delivery by emergency operative delivery that carries considerably more maternal risk than less urgent procedures. Neonatal outcomes are also significantly poorer following emergency caesarean for fetal distress [6].

Table 2 Relationship of cerebroplacental ratio (CPR) with different modes of deliveries

\begin{tabular}{|c|c|c|c|c|}
\hline \multirow[t]{2}{*}{ Variables } & \multirow[t]{2}{*}{ Total $(n=150)$} & \multicolumn{2}{|l|}{ CPR, $n(\%)$} & \multirow[t]{2}{*}{$p$ value } \\
\hline & & $\leq 1.1(n=25)$ & $>1.1(n=125)$ & \\
\hline \multicolumn{5}{|l|}{ Mode of delivery } \\
\hline Vaginal & $108(72)$ & $15(60)$ & $93(74.4)$ & $0.14^{\mathrm{a}}$ \\
\hline Cesarean section & $42(28)$ & $10(40)$ & $32(25.6)$ & \\
\hline Intrapartum fetal compromise & $7(16.7)$ & $4(40)$ & $3(9.4)$ & $0.043^{b}$ \\
\hline $\begin{array}{l}\text { Other causes (prolonged labor, cord prolapse, } \\
\text { malposition, tetanic uterus) }\end{array}$ & $35(83.3)$ & $6(60)$ & $29(90.6)$ & \\
\hline
\end{tabular}

CPR cerebroplacental ratio

${ }^{a} p$ values are based on chi-square test. Statistical significance at $p<0.05$

${ }^{\mathrm{b}} p$ values are based on Fisher's exact test. Statistical significance at $p<0.05$ 
Table 3 Relationship of cerebroplacental ratio (CPR) with neonatal outcomes

\begin{tabular}{|c|c|c|c|c|}
\hline \multirow[t]{2}{*}{ Variables } & \multirow[t]{2}{*}{ Total $(n=150)$} & \multicolumn{2}{|l|}{ CPR } & \multirow[t]{2}{*}{$p$ value } \\
\hline & & $\leq 1.1(n=25)$ & $>1.1(n=125)$ & \\
\hline Birth weight, mean $\pm S D$ & $3520.6 \pm 663.6$ & $3358 \pm 726.5$ & $3553.1 \pm 648.3$ & $0.25^{\mathrm{a}}$ \\
\hline Gestational age, mean \pm SD & $39.5 \pm 0.57$ & $39.7 \pm 0.67$ & $39.5 \pm 0.5$ & $0.09^{\mathrm{a}}$ \\
\hline \multicolumn{5}{|l|}{ Apgar score $<7$ at 1 min, $n(\%)$} \\
\hline Yes & $23(15.3)$ & $9(36)$ & $14(11.2)$ & \multirow[t]{2}{*}{$0.004^{c}$} \\
\hline No & $127(84.7)$ & $16(64)$ & $111(88.8)$ & \\
\hline \multicolumn{5}{|l|}{ Apgar score $<7$ at $5 \mathrm{~min}, n(\%)$} \\
\hline Yes & $8(5.3)$ & $5(20)$ & $3(2.4)$ & \multirow[t]{2}{*}{$0.003^{b}$} \\
\hline No & $142(94.7)$ & $20(80)$ & $122(97.6)$ & \\
\hline \multicolumn{5}{|l|}{ NICU admission } \\
\hline Yes & $19(12.7)$ & $8(32)$ & $11(8.8)$ & \multirow[t]{2}{*}{$0.004^{c}$} \\
\hline No & $131(87.3)$ & $17(68)$ & $114(91.2)$ & \\
\hline
\end{tabular}

${ }^{a} p$ values are based on Mann-Whitney test. Statistical significance at $p<0.05$

${ }^{\mathrm{b}} p$ values are based on chi-square test. Statistical significance at $p<0.05$

${ }^{c} p$ values are based on Fisher's exact test. Statistical significance at $p<0.05$

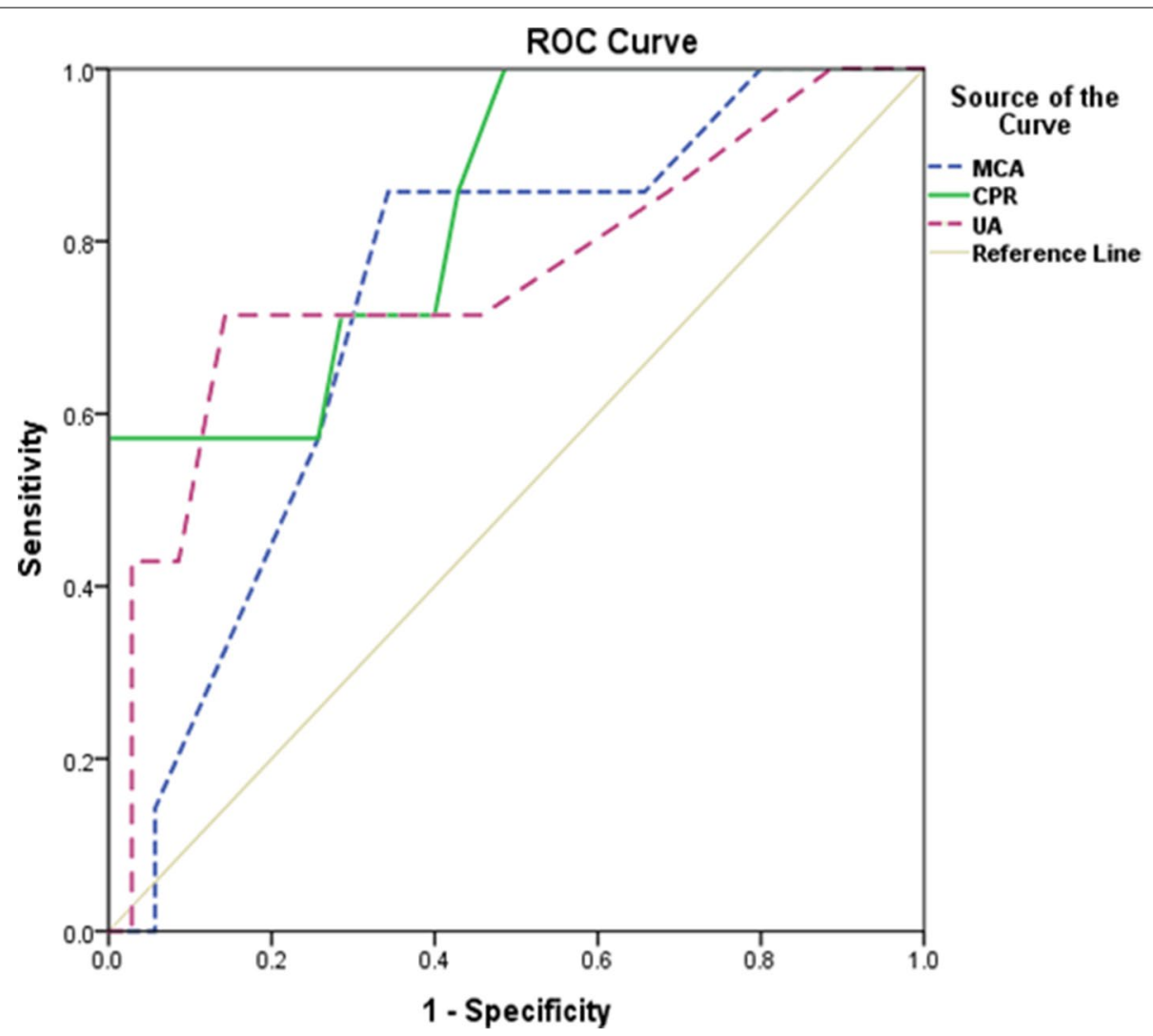

Fig. 2 Receiver-operating characteristics curves for prediction of cesarean section for intrapartum fetal compromise using cerebroplacental ratio, umbilical artery, and fetal middle cerebral artery pulsatility index 


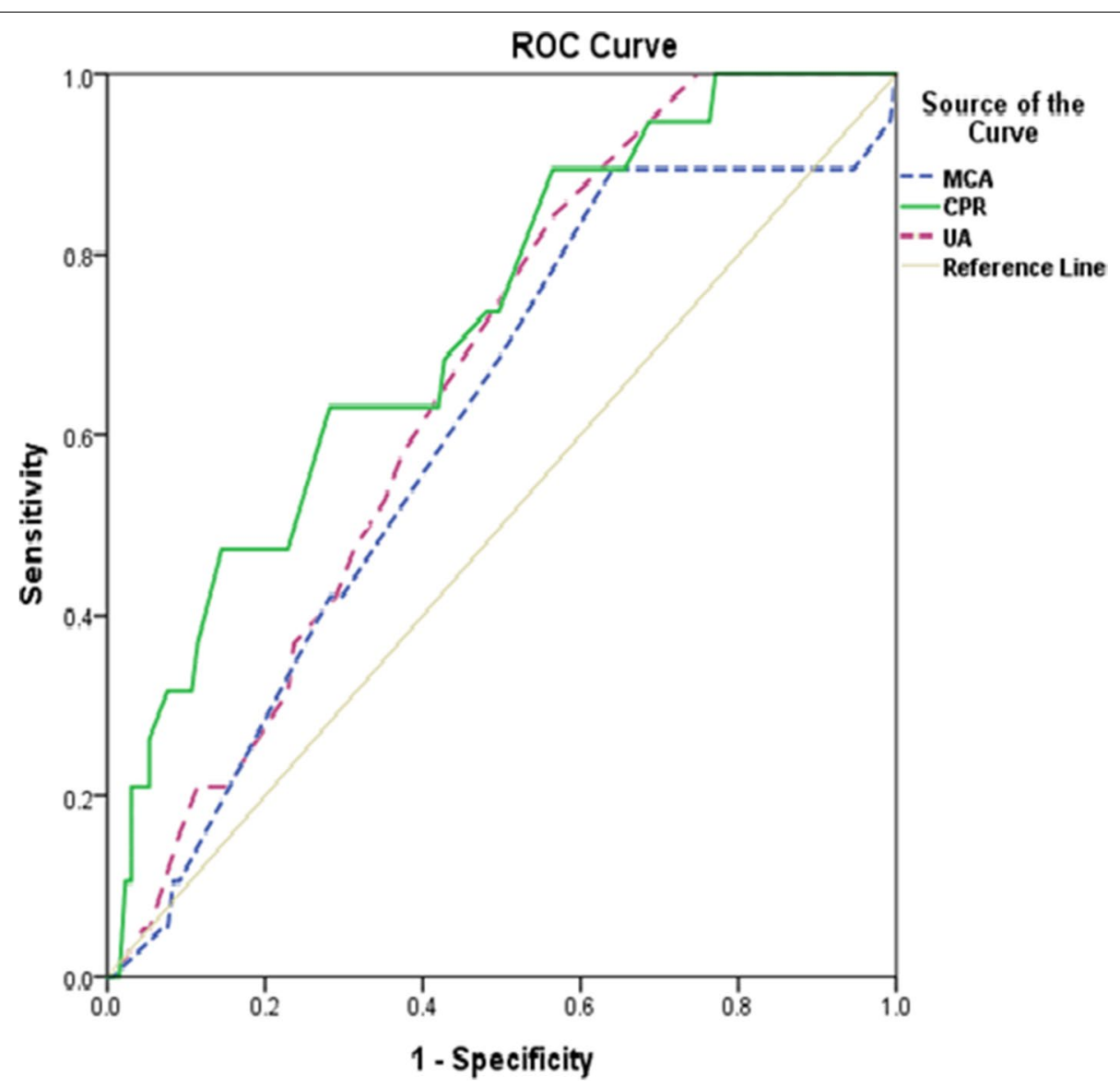

Fig. 3 Receiver-operating characteristic curves for prediction of NICU admission using cerebroplacental ratio, umbilical artery, and fetal middle cerebral artery pulsatility index

The most widely used screening method for intrapartum hypoxia is electronic fetal heart rate monitoring. It has very poor specificity and positive predictive value for hypoxia and adverse neonatal outcome [16].

Vulnerable fetuses often require rapid delivery by emergency cesarean section, which carries elevated maternal and fetal risks, and costs the healthcare system much more than vaginal delivery. The recent the UK Royal College of Obstetricians and Gynaecologists' Each Baby Counts Report reveals that $0.16 \%$ of all term babies born in 2015 died or suffered significant brain injury following the onset of labor. In conjunction with

Table 4 Sensitivity, specificity, PPV, NPV, and diagnostic accuracy of different sonographic parameters for prediction of adverse intrapartum outcomes

\begin{tabular}{|c|c|c|c|c|c|c|}
\hline Cut-off points & Cut of point & Sensitivity & Specificity & PPV* & NPV* & accuracy \\
\hline \multicolumn{7}{|l|}{ FHR abnormality by CTG } \\
\hline Umbilical artery PI & 1.005 & 63.89 & 68.42 & 38.89 & 85.71 & 67.33 \\
\hline Middle cerebral artery PI & 1.135 & 58.33 & 78.95 & 46.67 & 85.71 & 73.9 \\
\hline Cerebroplacental ratio & 1.205 & 63.89 & 76.32 & 46 & 87 & 73.3 \\
\hline \multicolumn{7}{|l|}{ CS delivery for IFC } \\
\hline Umbilical artery PI & 1.015 & 71.43 & 62.86 & 27.78 & 91.67 & 64.29 \\
\hline Middle cerebral artery PI & 1.25 & 85.71 & 65.71 & 33.33 & 95.83 & 69.05 \\
\hline Cerebroplacental ratio & 1.22 & 71.43 & 71.43 & 33.33 & 92.59 & 71.43 \\
\hline
\end{tabular}

PI pulsatility index, CS cesarean section, IFC intrapartum fetal compromise 
Table 5 Sensitivity, specificity, PPV, NPV, and diagnostic accuracy of different sonographic parameters for prediction of adverse neonatal outcomes

\begin{tabular}{|c|c|c|c|c|c|c|}
\hline Cut-off points & Cut of point & Sensitivity & Specificity & PPV* & NPV* & accuracy \\
\hline \multicolumn{7}{|l|}{ Apgar score $<7$ at $1 \mathrm{~min}$} \\
\hline Umbilical artery PI & 1.005 & 56.52 & 63.78 & 22.03 & 89.01 & 62.67 \\
\hline Middle cerebral artery PI & 1.25 & 78.3 & 52.8 & 23.08 & 93.06 & 56.57 \\
\hline Cerebroplacental ratio & 1.235 & 60.89 & 66.14 & 24.56 & 90.32 & 65.33 \\
\hline \multicolumn{7}{|l|}{ NICU admission } \\
\hline Umbilical artery PI & 1.005 & 57.89 & 63.36 & 18.64 & 91.21 & 62.67 \\
\hline Middle cerebral artery PI & 1.25 & 68.42 & 50.38 & 16.67 & 91.67 & 52.67 \\
\hline Cerebroplacental ratio & 1.205 & 63.16 & 70.99 & 24 & 93 & 70 \\
\hline
\end{tabular}

$P I$ pulsatility index, CS cesarean section, IFC intrapartum fetal compromise

other recommendations, this report highlights the need for continuous risk assessment commencing in the antenatal period and continuing intrapartum [17].

The current challenge is therefore to identify vulnerable fetuses at risk of hypoxic complications, particularly within an apparently low-risk cohort before the onset of labor.

The cerebroplacental ratio, a ratio of the middle cerebral artery PI and umbilical artery PI, is widely considered to be more indicative of adverse neonatal outcome than the assessment of blood flow indices individually [18].

We conducted this study aiming at assessing the fetal cerebroplacental ratio for prediction of adverse intrapartum and neonatal outcomes in a term, uncomplicated pregnancy.

One hundred fifty patients were included in the study, and participants were subjected to detailed history, physical examination, and ultrasound examination and were allocated into two groups according to CPR ratio with values below 1.1 as abnormal [19]. Afterwards, parameters characterizing the course of labor and the newborn's status were correlated with abnormal Doppler results.

The mean age of the participant's females was $29.2 \pm$ 4.1 years. More than half the sample was housewives (53.3\%). About $58.7 \%$ of the females came from rural areas and only $11.3 \%$ had a high socioeconomic status. About $72 \%$ had vaginal delivery while about $28 \%$ underwent cesarean section and $17 \%$ of them due to intrauterine fetal comprise.

Patients with CPR less than 1.1 were $16.7 \%$ and those with CPR more than 1.1 forms 83.3. There was no significant association between CPR with baseline and clinical characteristics of the pregnant females.

On assessing the association between rate of emergency cesarean section and CPR ratio, we found a significant increase in the prevalence of emergency cesarean sections in the group with CPR ratios $<1.1$ (40\% vs. 25.6\%). An abnormal CPR had a six-fold increased odds of cesarean section for inrapartum fetal compromise. The AUC values for cesarean sections for intrapartum fetal compromise (0.837) are considerably higher than those reported for intrapartum fetal heart rate monitoring $(0.60-0.63)[20]$.

In a subsequent study published 2015, Khalil et al. examined 8382 patients who underwent Doppler ultrasound examination after 37 weeks of gestation to evaluate the association between fetal cerebroplacental ratio (CPR) and intrapartum fetal compromise and admission to the neonatal unit, and their study showed that in fetuses requiring an emergency cesarean delivery for fetal distress, an abnormal CPR was significantly more frequent $(13.1 \%$ vs $9.4 \% ; P<.001)$ than those not requiring operative delivery [21].

Prior et al. prospectively evaluated 400 fetuses at term and reported that the CPR was shown to be an independent predictor of CS for intrapartum fetal compromise, and an abnormal CPR was found in $11 \%$. Of those who underwent cesarean delivery for fetal distress, $36.4 \%$ had an abnormal CPR compared with $10.1 \%(p<.001)$ that had a normal CPR. A low CPR ratio was associated with a six-fold increased rate of cesarean section for presumed fetal compromise. An abnormal CPR was a better predictor for an emergency cesarean delivery than an abnormal middle cerebral artery or umbilical artery [22].

In a study reported by Rosello et al., who evaluated the CPR in fetuses between 37 and 41.9 weeks of gestation, they found that the UA and venous $\mathrm{pH}$ were significantly lower in newborns who had an abnormal CPR than fetuses with a normal CPR. These data suggest that the CPR could be used to assess the risk of intrapartum fetal distress requiring cesarean delivery [23].

Another study conducted by Bligh et al. on 207 women with term uncomplicated pregnancy who studied the screening performance of low fetal cerebroplacental ratio and maternal placental growth factor level, for the prediction of cesarean section for intrapartum fetal 
compromise, reported that pregnancies delivered by CS for IFC had significantly lower mean CPR than the rest of the study cohort [24].

Women at a significant risk of IFC and/or adverse neonatal outcome could be offered elective birth when staff levels and availability of operative procedures are optimal. This could potentially reduce the number of emergency cesarean section procedures performed, thus improving maternal and neonatal outcomes.

In the current study, we found that infants with an abnormal CPR ratio had a significantly higher incidence of CTG abnormalities than those with a normal CPR ratio $(80 \%)$ vs $(12.8 \%)$, respectively, and that the likelihood of the having an abnormal FHR was increased more than twenty-seven-fold with abnormal CPR.

In the line of our study Ropaka et al. who studied the usefulness of Doppler velocimetry, based on cerebroplacental ratio evaluation, in predicting intrapartum fetal heart rate abnormalities and adverse neonatal outcome in 148 women with uncomplicated pregnancies at $40-42$ weeks reported that the percentage of abnormal CTG records in group with abnormal CPR (62.3\%) was significantly higher when compared to group with normal CPR (19.0\%) [25]. Prior et al. also reported that infants with a low CPR ratio had significantly higher rates of CTG abnormalities when compared with infants with a normal CPR ratio (82\% vs 31\%) [22].

We found that there was a significant relation between CPR and low Apgar score. Neonates with CPR $<1.1$ had significantly lower Apgar score at $1 \mathrm{~min}$ and $5 \mathrm{~min}$ than those with CPR $>1.1$ ( $36 \%$ vs $11.2 \%)$ and ( $20 \%$ vs 2.4 ), respectively. Pregnancies with $\mathrm{CPR}<1.1$ were 4.46 times more likely to have 1-min Apgar score $<7$ for neonates than those with CPR $>1.1$.

Prior et al. reported that Apgar scores $<7$ at both $1 \mathrm{~min}$ ( 56.5 vs $5.1 \%$ ) and 5 min (27.5 vs $1.3 \%$ ) were significantly lower with a low pre-labor CPR and described a greater frequency of poor Apgar scores in the low CPR group, but these did not reach significance [22].

Ropacka-Lesiak et al. also reported 1- and 5-min Apgar score to be significantly lower in patients with abnormal CPR when compared to the control group [25]. Meanwhile, Prior et al. 2013 reported no significant difference in the incidence of Apgar score $<7$ at 5 min between the CPR ratio percentile groups [22].

In addition, in this study, the rate of NICU admission was observed to be significantly higher in neonates with CPR $<1.1$ than those with CPR $<1.1$ (32 vs. $8.8 \%$ ) respectively, and that the odds of being admitted to NICU is 4.87 times greater for neonates who have CPR $\leq 1.1$ vs. those who have CPR $>1.1$.

In the line of our study, Khalil et al. reported the rate of NICU admission to be higher in group with abnormal
CPR $14.3 \%$ vs 9.7\%, while Prior 2013 described no significant difference in the rate of neonatal unit admission between the CPR ratio percentile groups [21].

Our result suggested that the cut-off value of CPR 1.1 is a reliable value to predict adverse intrapartum and neonatal outcomes, which is consistent with the value of 1.1 recommended by Arbeille et al. and DeVore.

Evaluation of the performance of CPR revealed that it has $(63 \%)$ sensitivity, (71\%) specificity, and (70\%) accuracy, and the positive and negative predictive values were (24) and (93), respectively.

Previous studies on CPR have provided conflicting reports on its test performance. Murata et al. found that CPR with cut-off value of 1.1 had $62 \%$ sensitivity and $74.5 \%$ specificity to predict adverse outcome [26]. Prior et al. reported that a low CPR is associated with increased risk of emergency operative delivery for IFC (sensitivity $32.5 \%$, specificity $93.2 \%$, and positive predictive value $36.4 \%$ ) [22]. Devine et al. obtained contrasting results. They suggested CPR values below 1.05 to be a good predictor of adverse perinatal outcome in postterm pregnancies, but the study included a number of high-risk pregnancies complicated by hypertension or gestational diabetes [27].

In contrast, Bakalis et al. reported using retrospective data in an unselected population, CPR measured at 30-34 weeks' gestation was shown to be a weak predictor of perinatal complications, with detection rates of $5-11 \%$ and $20-50 \%$ when measured $>2$ weeks and $<2$ weeks from delivery, respectively [28].

There are few reports regarding the screening efficiency of the cut-off value of CPR. It may be difficult to determine the fixed cut-off value of CPR for screening because CPR changes depending on gestational ages during pregnancy. Taken into consideration, the reports that summarize the data by Baschat and Genbruch, our cut-off value of CPR 1.1 seems to be appropriate for clinical application in term uncomplicated pregnancy [29]. Some authors suggest that reference ranges should be applied for the CPR assessment. However, there are studies which revealed no superiority of reference ranges over cut-off values with regard to adverse perinatal outcome prediction [30, 31].

Gramellini, as well as other researchers, reported that CPR values are constant throughout the last 10 weeks of pregnancy. That is why the value of 1.1 was established as a cutoff point, and values $<1.1$ were reported as abnormal [32].

The current study showed that the cerebroplacental ratio had a sensitivity of (63\%) which represents an advance on current practice, in which up to $60 \%$ of births complicated by intrapartum compromise occur in pregnancies with no apparent antenatal risk factors [2]. 


\section{Conclusions}

The cerebroplacental ratio shows the highest sensitivity in the prediction of fetal heart rate abnormalities and adverse neonatal outcome in uncomplicated pregnancies at term. The cerebroplacental ratio index is useful in clinical practice in antenatal monitoring of these women in order to select those at high risk of intra- and postpartum fetal complications (Fig. 3).

Cerebroplacental ratio can predict women at significant risk of intrapartum fetal compromise and/or adverse neonatal outcome, and those could be offered elective birth. This could potentially reduce the number of emergency cesarean section procedures performed, thus improving maternal and neonatal outcomes.

\section{Abbreviations}

AUC: Area under the curve; CPR: Cerebroplacental ratio; CS: Cesarean section: CTG: Cardiotocography; FHR: Fetal heart rate; IFC: Intra-partum fetal compromise; MCA: Middle cerebral artery; MCA PI: Middle Cerebral Artery Pulsatility Index; MCA RI: Middle Cerebral Artery Resistance Index; NICU: Neonatal intensive care unit; PI: Pulsatility index; UA: Umbilical artery; UA PI: Umbilical Artery Pulsatility Index; UA RI: Umbilical Artery Resistance Index.

\section{Acknowledgements}

Special thanks, admiration, and respect to all our department members for their kind help and valuable support.

\section{Authors' contributions}

ML participated in the design, methodological issues, conduct, analysis, interpretation of the study, and scientific writing. SA participated in the data collection, performed the ultrasound examination, follow-up of the cases, stastical analysis, and scientific writing. AM is the reviewer and supervisor of the study. The authors have written, seen and approved the final version of the manuscript.

\section{Funding}

No funding resources.

\section{Availability of data and materials}

The datasets used and/or analyzed during the current study are available from the corresponding author on reasonable request.

\section{Declarations}

\section{Ethical approval and consent to participate}

All procedures performed on our study involving human participants were in accordance with the ethical standards of the institutional and/or national research committee. This research has been reviewed by our research ethics commie in the Faculty of Medicine of Suez Canal University. Written and verbal consent was obtained from the selected patients. Ethical committee reference number is not available.

\section{Consent for publication}

Not applicable.

\section{Competing interests}

The authors declare that they have no competing interests.

Received: 29 March 2021 Accepted: 2 December 2021

Published online: 14 December 2021

\section{References}

1. Blencowe H, Cousens S, Jassir FB, Say L, Chou D, Mathers C, Lawn JE (2016) National, regional, and worldwide estimates of stillbirth rates in 2015, with trends from 2000: a systematic analysis. Lancet Glob Health 4(2):e98-e108

2. Low JA, Pickersgill H, Killen H, Derrick EJ (2001) The prediction and prevention of intrapartum fetal asphyxia in term pregnancies. Am J Obstet Gynecol 184(4):724-730

3. Bligh LN, Alsolai AA, Greer RM, Kumar S (2017) Cerebroplacental ratio thresholds measured within 2 weeks before birth and risk of cesarean section for intrapartum fetal compromise and adverse neonatal outcome. Ultrasound Obstet Gynecol 52(3):340-346

4. Bardien N, Whitehead CL, Tong S, Ugoni A, McDonald S, Walker SP (2016) Placental insufficiency in fetuses that slow in growth but are born appropriate for gestational age: a prospective longitudinal study. PLOS ONE 11(1):e0142788

5. Mclntyre S, Taitz D, Keogh J, Goldsmith S, Badawi N, Blair EVE (2013) A systematic review of risk factors for cerebral palsy in children born at term in developed countries. Dev Med Child Neurol 55(6):499-508

6. Grace L, Greer RM, Kumar S (2015) Perinatal consequences of a category 1 caesarean section at term. BMJ Open 5(7):e007248-e007248

7. Ropacka-Lesiak M, Korbelak T, Bręborowicz G (2012) Hypoxia index in the prediction of abnormal CTG at delivery in uncomplicated pregnancies. Neuroendocrinol Lett 33:5-9

8. Scherjon S, Briët J, Oosting H, Kok J (2000) The discrepancy between maturation of visual-evoked potentials and cognitive outcome at five years in very preterm infants with and without hemodynamic signs of fetal brainsparing. Pediatrics 105(2):385-391

9. Parer JT, King TL (2011) Electronic fetal monitoring as a public health screening program: the arithmetic of failure to the editor. Obstet Gynecol 117(4):986

10. KorbelakT, Ropacka-Lesiak M, Bręborowicz GH (2012) Doppler blood flow velocimetry in the umbilical artery in uncomplicated pregnancy. Ginekologia Polska 83(1):38-45

11. Cruz-Martinez R, Figueras F, Hernandez-Andrade E, Oros D, Gratacos E (2011) Fetal brain Doppler to predict cesarean delivery for nonreassuring fetal status in term small-for-gestational-age fetuses. Obstet Gynecol 117(3):618-626

12. Arbeille P, Carles G, Chevillot M, Locatelli A, Herve P, Perrotin F, Maulik D (2005) Cerebral and umbilical doppler in the prediction of fetal outcome. Doppler Ultrasound Obstet Gynecol 121;177-197

13. Dunn L, Sherrell H, Kumar S (2017) Review: systematic review of the utility of the fetal cerebroplacental ratio measured at term for the prediction of adverse perinatal outcome. Placenta 54:68-75

14. Khalil A, Morales-Rosello J, Townsend R, Morlando M, Papageorghiou A, Bhide A, Thilaganathan B (2016) Value of third-trimester cerebroplacental ratio and uterine artery Doppler indices as predictors of stillbirth and perinatal loss. Ultrasound Obstet Gynecol 47(1):74-80

15. Hilder L, Zhichao Z, Parker M, Jahan S, Chambers GM (2014) Australia's mothers and babies 2012. Perinat Stat Ser 30:69

16. Grimes DA, Peipert JF (2010) Electronic fetal monitoring as a public health screening program: the arithmetic of failure. Obstet Gynecol 116(6):1397-1400

17. Royal College of Obstetricians \& Gynaecologists. Each baby counts: key messages from 2015. https://www.rcog.org.uk/globalassets/documents/ guidelines/research\%2D\%2Daudit/rcog-each-baby-counts-report.pdf

18. Hershkovitz R, Kingdom JCP, Geary M, Rodeck CH (2000) Fetal cerebral blood flow redistribution in late gestation: identification of compromise in small fetuses with normal umbilical artery Doppler. Ultrasound Obstet Gynecol 15(3):209-212

19. DeVore GR (2015) The importance of the cerebroplacental ratio in the evaluation of fetal well-being in SGA and AGA fetuses. Am J Obst Gynecol 213(1):5-15

20. Di Tommaso M, Seravalli V, Cordisco A, Consorti G, Mecacci F, Rizzello F (2013) Comparison of five classification systems for interpreting electronic fetal monitoring in predicting neonatal status at birth. J Maternal-Fetal Neonat Med 26(5):487-490

21. Khalil AA, Morales-Rosello J, Morlando M, Hannan H, Bhide A, Papageorghiou A, Thilaganathan B (2015) Is fetal cerebroplacental ratio an 
independent predictor of intrapartum fetal compromise and neonatal unit admission? Am J Obstet Gynecol 213(1):54.e1

22. Prior T, Mullins E, Bennett P, Kumar S (2013) Prediction of intrapartum fetal compromise using the cerebroumbilical ratio: a prospective observational study. Am J Obstet Gynecol 208(2):124-1e1

23. Morales-Roselló J, Khalil A, Morlando M, Bhide A, Papageorghiou A, Thilaganathan B (2015) Poor neonatal acid-base status in term fetuses with low cerebroplacental ratio. Ultrasound Obst Gynecol 45(2):156-161

24. Bligh LN, Alsolai AA, Greer RM, Kumar S (2018) Prelabor screening for intrapartum fetal compromise in low-risk pregnancies at term: cerebroplacental ratio and placental growth factor. Ultrasound Obstet Gynecol 52(6):750-756

25. Ropacka-Lesiak M, Korbelak T, Świder-Musielak J, Breborowicz G (2015) Cerebroplacental ratio in prediction of adverse perinatal outcome and fetal heart rate disturbances in uncomplicated pregnancy at 40 weeks and beyond. Arch Med Sci 11(1):142

26. Murata S, Nakata M, Sumie M, Sugino N (2011) The Doppler cerebroplacental ratio predicts non-reassuring fetal status in intrauterine growth restricted fetuses at term. J Obstet Gynaecol Res 37(10):1433-1437

27. Devine PA, Bracero LA, Lysikiewicz A, Evans R, Womack S, Byrne DW (1994) Middle cerebral to umbilical artery Doppler ratio in post-date pregnancies. Obstet Gynecol 84(5):856-860

28. Bakalis S, Akolekar R, Gallo DM, Poon LC, Nicolaides KH (2015) Umbilical and fetal middle cerebral artery Doppler at 30-34 weeks' gestation in the prediction of adverse perinatal outcome. Ultrasound Obstet Gynecol 45(4):409-420

29. Baschat AA, Gembruch U (2003) The cerebroplacental Doppler ratio revisited. Ultrasound Obstet Gynecol 21(2):124-127

30. Ebbing C, Rasmussen S, Kiserud T (2007) Middle cerebral artery blood flow velocities and pulsatility index and the cerebroplacental pulsatility ratio: longitudinal reference ranges and terms for serial measurements. Ultrasound Obstet Gynecol 30(3):287-296

31. Odibo AO, Riddick C, Pare E, Stamilio DM, Macones GA (2005) Cerebroplacental Doppler ratio and adverse perinatal outcomes in intrauterine growth restriction: evaluating the impact of using gestational age-specific reference values. J Ultrasound Med 24(9):1223-1228

32. Gramellini D, Folli MC, Raboni S, Vadora E, Merialdi A (1992) Cerebralumbilical Doppler ratio as a predictor of adverse perinatal outcome. Obstet Gynecol 79(3):416-420

33. Simiyu IN, Mchaile DN, Katsongeri K, Philemon RN, Msuya SE (2017) Prevalence, severity and early outcomes of hypoxic ischemic encephalopathy among newborns at a tertiary hospital, in northern Tanzania. BMC Pediatr 17(1):131

\section{Publisher's Note}

Springer Nature remains neutral with regard to jurisdictional claims in published maps and institutional affiliations.

\section{Submit your manuscript to a SpringerOpen ${ }^{\circ}$ journal and benefit from:}

- Convenient online submission

- Rigorous peer review

- Open access: articles freely available online

- High visibility within the field

- Retaining the copyright to your article 